Ann. Biol. anim. Bioch. Biophys., I972, 12 (r), r49-I57.

\title{
INFLUENCE DES FACTEURS LIPOTROPES SUR LE MÉTABOLISME DU CHOLESTÉROL CHEZ LE CANETON ${ }^{(1)}$
}

\author{
J.-L. SAHEB et J.-M. DEMERS* \\ Institut de Diététique et de Nutrition, \\ Faculté de Médecine, Université de Montréal \\ *Département de Biologie, \\ Faculté des Sciences, Université de Montréal
}

\section{RÉSUMÉ}

Dans le but d'étudier le rôle de certains facteurs lipotropes (choline et inositol) et celui de certaines vitamines (pyridoxine et acide pantothénique) dans l'estérification du cholestérol chez le caneton, nous avons soumis IOz oiseaux, au cours de deux séries d'expériences, à des régimes carencés successivement en chacune de ces quatre substances. Ceci nous a permis d'observer les effets qui se sont manifestés sur la croissance, la cholestérolémie, le cholestérol musculaire, hépatique et cardiaque tant sous sa forme libre que sous sa forme estérifiée, et enfin sur le niveau des lipides totaux du muscle, du foie et du cœur.

En résumé, les résultats obtenus nous permettent de conclure ce qui suit :

I. dans les conditions expérimentales décrites (régime à $25 \mathrm{p}$. roo de protéines et I,5 p. Ioo de méthionine), la choline n'est pas essentielle à la prévention de la stéatose hépatique mais demeure un facteur de croissance important et est nécessaire pour prévenir la pérose;

2. un déficit en choline n'affecte pas la cholestérolémie mais augmente le cholestérol du muscle et du cœur, alors qu'un déficit en pantothénate diminue considérablement la cholestérolémie et augmente le cholestérol musculaire;

3. un déficit en pyridoxine, malgré un effet néfaste sur la croissance, ne semble pas affecter le métabolisme du cholestérol et des lipides en général ;

4. autant qu'il est pəssible d'en juger par le rapport cholestérol estérifié/cholestérol total du muscle, du foie et du cœur, une carence en choline ou en pantothénate semble être reliée à une activité accrue du plasma dans l'estérification du cholestérol.

\section{INTRODUCTION}

SPERRy (I935) a été le premier à montrer que le sang du rat contient une diastase pouvant estérifier le cholestérol. Cet enzyme, une acyl-transférase, est fort probablement produit dans le foie (BROT et al., I962) mais sa synthèse serait influencée

(') Travail exécuté grâce à un subside du Conseil National des Recherches du Canada. 
par l'état nutritionnel de l'animal, puisque les esters de cholestérol synthétisés dans le foie de l'animal soumis au jeûne diffèrent de ceux de l'animal bien nourri (SwELI. et LAw, I967). De plus, il semble que le sang des oiseaux renferme plusieurs transférases de ce type (Sugano et al., I966). D'autre part, en I965, WELLS et BUCKLEY (1965) ont observé pour la première fois qu'un facteur lipotrope, la choline, pouvait influencer l'estérification du cholestérol. Ces auteurs ont en effet remarqué que des rats privés de choline, excrètent dans leur bile du cholestérol libre en quantité égale à celle excrétée par des rats normaux, mais pas du tout d'esters de cholestérol. Des facteurs lipotropes autres que la choline peuvent aussi influencer l'estérification du cholestérol (WELLs et HoGaN, I968).

Étant intéressés depuis de nombreuses années par les facteurs lipotropes et ayant étudié chez le caneton le métabolisme du cholestérol (ALARY et DEMERS, I965; Carbonneau et Demers, I $965 b$ ), nous avons pensé qu'il serait intéressant d'étudier le rôle de certains facteurs lipotropes et de certaines vitamines dans la répartition du cholestérol libre et estérifié dans les différents organes du caneton, l'objet du présent travail, et éventuellement son estérification au niveau du sang.

\section{TECHNIQUE}

Des canetons de race Pékin (1) âgés d'un jour au moment de leur réception, ont été utilisés pour ces expériences. Dès leur arrivée, les oiseaux ont été alimentés avec un régime commer-

TABLEAU I

Composition centésimale du régime de base

\begin{tabular}{|c|c|}
\hline Ingrédients & P. 100 \\
\hline Riz décortiqué moulu ( $\left.{ }^{1}\right)$ & 57,9 \\
\hline Cellulose $\ldots \ldots \ldots \ldots \ldots \ldots \ldots \ldots \ldots \ldots$ & 4 \\
\hline Caséine $\left({ }^{2}\right)(3,5$ p. 100 de méthionine $) \ldots \ldots \ldots \ldots \ldots \ldots$ & 19 \\
\hline Huile de mais $\left({ }^{(s)}, \ldots \ldots \ldots \ldots \ldots \ldots \ldots \ldots \ldots \ldots \ldots\right.$ & 12 \\
\hline Sels minéraux $\left.{ }^{4}\right) \quad \ldots \ldots \ldots \ldots \ldots \ldots \ldots \ldots \ldots \ldots$ & 5 \\
\hline 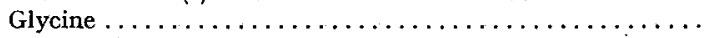 & 1 \\
\hline DL-méthionine $\ldots \ldots \ldots \ldots \ldots \ldots \ldots \ldots \ldots \ldots$ & 0,8 \\
\hline Chlorure de choline $\ldots \ldots \ldots \ldots \ldots \ldots \ldots \ldots \ldots \ldots$ & 0,3 \\
\hline Mélange vitaminique $\left.{ }^{5}{ }^{5}\right) \ldots \ldots \ldots \ldots \ldots \ldots \ldots \ldots$ & 0,3 \\
\hline
\end{tabular}

(1) Rice Flour, Mount Royal Rice Mills, Montréal (7 p. 100 protéine).

$\left({ }^{2}\right)$ Vitamin Free Casein, Nutritional Biochemicals Corp., Cleveland.

(3) Mazola: The Canada Starch Co. Ltd., Montréal-Toronto. land.

(4) Salt mixture, "Hegsted ", Nutritional Biochemicals Corp., Cleve-

$\left.{ }^{5}\right)$ Vitamines $\mathrm{mg} / 100 \mathrm{~g}$ de régime : acide folique 0,2 ; chlorhydrate de thiamine 0,4 ; chlorhydrate de pyridoxine 0,4 ; riboflavine 0,8 ; pantothénate de calcium 2,5 ; acide nicotinique 0,4 ; acide $p$-amino benzoïque 0,2 ; vitamine $\mathrm{B}_{12} 0,0036$; biotine 0,$02 ; \alpha$-tocophérol 10,$0 ; 2$-méthyl naphtoquinone 1,0 ; vitamine A $4500 \mathrm{UI}$; vitamine D $600 \mathrm{UI}$.

(') Canetons mâles provenant de Webfoot Farm and Hatchery Ltd., Elora, Ontario. 
cial (1) pendant deux jours. Cette étape pré-expérimentale est obligatoire a fin d'éviter le taux élevé de mortalité observé chez les oiseaux lorsqu'ils reçoivent dès le début les régimes purifiés (HEGSTED et Stare, 1945). Pour chacune des deux expériences $\left({ }^{2}\right)$, les canetons, dont le poids moyen au moment de la mise en expérience était respectivement de $70 \mathrm{~g}$ et de $90 \mathrm{~g}$, ont été répartis en lots de neuf. Seul le lot témoin (régime commercial) était constitué de six animaux. Pesés tous les deux jours, les canetons étaient nourris et abreuvés ad libitum.

Au cours de ces travaux nous avons étudié l'effet de cinq régimes expérimentaux provenant de la soustraction successive du régime de base (tabl. I), de certains facteurs lipotropes (choline et inositol) et de certaines vitamines (pyridoxine et acide pantothénique). Cette soustraction a été opérée au profit de la farine de riz.

Au bout de 2 I jours, les canetons ont été soumis à un jeûne de seize heures avant d'être sacrifiés. Immédiatement avant l'abattage, nous avons prélevé du sang par ponction cardiaque selon la technique de Genest (1946). Le sérum obtenu par coagulation et centrifugation a servi au dosage du cholestérol sérique selon la méthode de PEARson et al. (1954), ainsi qu'à la détermination de la lipémie selon la méthode de SwaHN (1952). Le foie, le cœur ainsi qu'un échantillon des muscles de la cuisse, ont été prélevés a fin d'y doser le cholestérol selon une modification de la méthode de PEARson et al. (I954), ainsi que les lipides totaux, selon une modification de la technique de Folch et al. (1951; r954).

\section{RÉSULTATS}

\section{I. - Croissance (tab1. 2)}

Les groupes privés de choline ont subi un retard considérable dans leur croissance. Ce retard est significatif $(p<0,0 I)$ comparé aux groupes recevant le régime de base qui contient 0,03 p. Ioo de chlorure de choline. De plus, tous les oiseaux qui

\section{TABLEAU 2}

Croissance et lipides musculaires, hépatiques et cardiaques de canetons soumis à des régimes pauvres en facteurs lipotropes

\begin{tabular}{|c|c|c|c|c|c|}
\hline \multirow{2}{*}{$\begin{array}{l}\text { Soustraction } \\
\text { régime de base }\end{array}$} & \multicolumn{2}{|c|}{ Gain pondéral (g) 21 jours } & \multicolumn{3}{|c|}{ Lipides p. $100 \mathrm{du}$ poids frais } \\
\hline & Expérience $\mathrm{A}$ & Expérience B & Muscle & Foie & Cœur \\
\hline 一 & $499 \pm 57$ & $459 \pm 33$ & $1,99 \pm 0,11$ & $3,32 \pm 0,20$ & $3,99 \pm 0,19$ \\
\hline Choline ... & $256 \pm 20^{* *}$ & $338 \pm 40 * *$ & $2,37 \pm 0,15$ & $4,15 \pm 0,43$ & $4,42 \pm 0,16(1)$ \\
\hline Inositol & $447 \pm 27$ & $418 \pm 28$ & $2,01 \pm 0,11$ & $3,17 \pm 0,19$ & $4,62 \pm 0,21 *$ \\
\hline Pyridoxine. . & $280 \pm 32 * *$ & $243 \pm 30^{* *}$ & $2,22 \pm 0,18$ & $3,70 \pm 0,35$ & $3,16 \pm 0,25 *$ \\
\hline Pantothénate .... & $343 \pm 28^{* *}$ & $353 \pm 20^{* *}$ & $2,39 \pm 0,16$ & $3,87 \pm 0,31$ & $3,95 \pm 0,20$ \\
\hline Commercial & $705 \pm 57$ & $649 \pm 47$ & $2,16 \pm 0,23$ & $3,67 \pm 0,09$ & $4,07 \pm 0,17$ \\
\hline
\end{tabular}

\pm Erreur standard à la moyenne $=\mathrm{S} \bar{x}=\sqrt{\frac{\sum\left(x_{1}-\bar{x}\right)^{2}}{n(n-1)}}$

* $P=<0,05$ vs régime de base.

** $\mathrm{P}=<0,01$ vs régime de base.

(1) Mélange alimentaire pré-début pour poussins (Poussex : 21 p. Ioo de protéines).

(2) Expérience A (début de l'été) et expérience B (fin de l'été : éclosion plus tardive, oiseaux ordinairement moins vigoureux). Les oiseaux de l'expérience $B$ ont surtout servi pour l'étude des variations du cholestérol musculaire, hépatique et cardiaque. 
en sont privés ont présenté des signes évidents de pérose unilatérale ou bilatérale. La croissance est aussi retardée de façon significative $(p<0,0 I)$ chez les canetons pour lesquels on a soustrait la pyridoxine ou le pantothénate de calcium du régime de base. Par contre, l'absence d'inositol dans le régime ne semble pas affecter la croissance.

\section{2. - Lipides musculaires, hépatiques et cardiaques (tab1. 2)}

Il y a une légère augmentation des lipides totaux dans le muscle, le foie et le cour des animaux privés de choline. Cette augmentation n'est toutefois pas significative par rapport aux groupes recevant le régime de base. On remarque aussi une augmentation non significative des lipides totaux du muscle et du foie chez les oiseaux carencés en pantothénate de calcium. Par contre, il y a augmentation significative $(p<0,05)$ des lipides cardiaques chez les animaux auxquels on a retiré l'inositol dans leur régime, mais diminution $(p<0,05)$ des mêmes lipides chez les animaux privés de pyridoxine.

\section{3. - Cholestérolémie (tabl. 3)}

On trouve moins de cholestérol total $(p<0,0$ I) et de cholestérol estérifié $(p<0,05)$ dans le sérum des canetons carencés en pantothénate que dans tous les autres groupes. C'est dans les groupes privés de choline que l'on observe la cholestérolémie la plus élevée, toutefois cette élévation n'est pas significative par rapport aux groupes recevant le régime de base. Quant au rapport cholestérol estérifié/cholestérol total, il est élevé dans tous les groupes expérimentaux sauf dans ceux recevant le régime de base et ceux carencés en pyridoxine.

\section{4. - Cholestérol musculaire, hépatique et cardiaque (tabl. 3)}

\section{a) Cholestérol musculaire.}

Chez les animaux carencés en choline il y a, dans le muscle, une augmentation significative du cholestérol total $(p<0,0$ ) $)$ et du cholestérol estérifié $(p<0,05)$ par rapport aux oiseaux recevant le régime de base. La même observation peut être faite pour les canetons privés de pantothénate. En ce qui concerne les groupes privés de pyridoxine, l'augmentation significative $(p<0,05)$ du cholestérol musculaire ne s'observe que dans le cas du cholestérol total. Malgré ces augmentations, parfois considérables, le rapport cholestérol estérifié/cholestérol total n'est pas modifié chez les animaux soumis aux régimes expérimentaux, sauf dans le cas du groupe privé de pantothénate où ce rapport est nettement plus élevé $(0,90)$. Enfin, il faut noter que le cholestérol estérifié par rapport au cholestérol total est encore plus élevé chez les oiseaux alimentés au régime commercial $(0,93)$.

\section{b) Cholestérol hépatique.}

Seuls les groupes privés d'inositol ont présenté un cholestérol hépatique plus élevé par rapport aux groupes nourris au régime de base. Encore cette élévation n'est-elle significative $(p<0,0$ I) que pour le cholestérol total. Quant au rapport cholestérol estérifié/cholestérol total, il est beaucoup plus élevé dans les groupes 


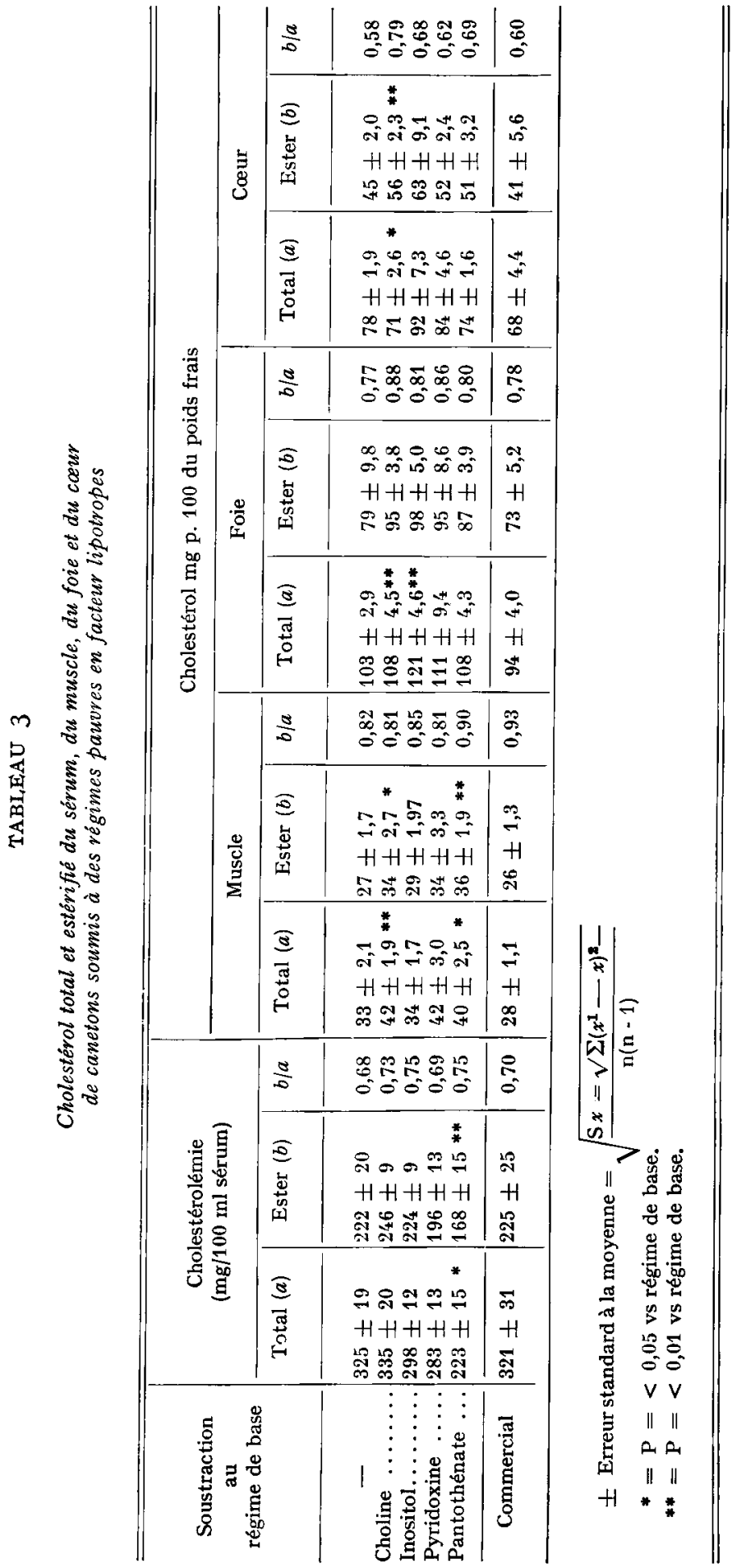


privés de choline et dans ceux privés de pyridoxine. Ceci dépend plus d'une élévation du cholestérol estérifié que d'une baisse du cholestérol total.

\section{c) Cholestérol cardiaque.}

C'est encore dans les groupes privés de choline que l'on observe les variations les plus importantes du cholestérol cardiaque. Alors qu'il y a, par rapport aux groupes témoins (régime de base), diminution significative du cholestérol cardiaque total $(p<0,05)$, il y a au contraire, augmentation significative du cholestérol estérifié $(p<0,05)$. Il s'ensuit que le rapport cholestérol estérifié sur cholestérol total passe de $0,58 \mathrm{chez}$ les témoins (régime de base) à $0,78 \mathrm{chez}$ les oiseaux carencés en choline. Ce même rapport est aussi plus élevé chez les canetons carencés soit en inositol $(0,68)$, soit en panthoténate $(0,69)$.

\section{DISCUSSION}

La choline, encore une fois (CARBONNEAU et DEMERS, I950), s'avère un facteur de croissance important pour le caneton et est nécessaire pour la prévention de la pérose. Il n'y a pas lieu d'être surpris de l'absence d'infiltration grasse hépatique chez les oiseaux privés de choline. Comme nous l'avons déjà montré (CARBonNEAU et DEMERS, I965 $a$ ), un déficit de choline dans le régime n'entraîne pas nécessairement une stéatose hépatique chez le caneton. Quand l'animal reçoit une alimentation riche en protéines et en méthionine, comme c'est le cas dans les expériences présentes, il n'y a pas de stéatose hépatique chez le caneton pourvu qu'existent, dans le régime, des groupements méthyles labiles.

Un déficit d'inositol ne semble pas avoir beaucoup d'effets sur la répartition des lipides et du cholestérol des différents tissus de l'animal, si ce n'est une hausse du cholestérol total du foie de même qu'une augmentation moins importante du cholestérol total et estérifié du cœur, Nos résultats rejoignent en ceci ceux de SNYDER et al. (I957) sans pour cela que nous puissions dire que l'inositol influence l'activité de l'acyl-transférase du plasma. Toutefois, nous tenons à faire remarquer que le rapport cholestérol libre-cholestérol estérifié dans le plasma est fortement abaissé dans le cas d'un déficit en inositol exactement comme dans les travaux de WEILs et HoGAN (Ig68) chez le rat.

Ces auteurs (WELLs et HoGAN, I968) ont remarqué une baisse de la cholestérolémie chez les rats expérimentaux (privés des mêmes vitamines que nos canetons) par rapport aux animaux nourris au régime commercial. Le cholestérol, sous sa forme libre, est fortement diminué chez les rats privés soit de pyridoxine, soit d'inositol. Il s'ensuit une diminution considérable du rapport cholestérol libre/cholestérol estérifié chez les rats de ces deux groupes. Dans nos expériences, nous obtenons des résultats concordants en ce qui concerne la cholestérolémie totale, sauf pour les oiseaux privés de choline. Cependant, pour ce qui est du rapport cholestérol libre/ cholestérol estérifié, il est abaissé surtout dans le cas d'une carence en inositol, en pantothénate et en choline, mais n'est pas affecté lors d'une privation de pyridoxine. 
Par ailleurs, chez les canetons privés de choline, il nous faut souligner que la très forte augmentation des esters de cholestérol dans le muscle, le foie et le cœur, alors que le cholestérol libre dans les mêmes organes reste à peu près stable, semble être reliée à une activité accrue de l'acyl-transférase du plasma. La même remarque pourrait être faite pour les oiseaux privés de pantothénate. Nous n'avons aucune preuve directe de cette liaison, puisque nous n'avons pas étudié le taux d'estérification du cholestérol dans le plasma, mais nous nous référons ici aux travaux de WELLS et HogAN (I968) qui ont obtenu des résultats semblables chez le rat. Ces auteurs ont remarqué, en effet, que de fortes concentrations d'esters de cholestérol dans le foie, le muscle et le cœur, accompagnées d'un niveau de cholestérol libre stable, se retrouvaient chaque fois que l'activité de 1'acyl-transférase plasmatique était augmentée.

On ne reconnaît pas encore très bien la source des esters de cholestérol des différents tissus chez les mammifères et les oiseaux. FIELD et al. (I960) ont bien remarqué chez l'homme que le cholestérol des tissus, aussi bien sous sa forme libre que sous sa forme estérifiée, provient du cholestérol plasmatique. Toutefois, d'autres auteurs (LoFLAND et al., 1965) ont montré que l'estérification du cholestérol peut aussi se faire dans les tissus mêmes, comme c'est le cas pour l'aorte du pigeon au cours de 1'athérogenèse.

En se basant sur les travaux de WELLS et HOGAN (I968), il est permis de penser que l'augmentation des esters du cholestérol dans les tissus, observée surtout chez les canetons carencés en choline ou en pantothénate, provient probablement du plasma. Les esters s'accumuleraient dans les tissus par suite d'une plus forte estérification du cholestérol au niveau du plasma due à une augmentation de l'acyltransférase dans cette humeur (SHAH et al., 1964).

Pour connaître la provenance des esters du cholestérol des tissus, il faudrait, au moyen d'éléments marqués, faire l'analyse des acides gras des esters de cholestérol du sang et des tissus, et sur les mêmes animaux évaluer le niveau d'estérification du cholestérol dans le plasma. C'est ce qui fera l'objet d'une prochaine étude.

\section{CONCLUSION}

L'étude des effets de certains facteurs lipotropes et de certaines vitamines sur l'estérification du cholestérol effectuée chez le caneton, nous conduit à penser qu'il existe une relation possible entre une carence en choline ou en pantothénate et une activité accrue du plasma dans l'estérification du cholestérol. Cette conclusion peut être tirée de l'examen du rapport cholestérol estérifié/cholestérol total du muscle, du foie et du cœur obtenu chez des canetons soumis à des régimes respectivement carencés en chacune des substances suivantes : choline, inositol, pyridoxine et acide pantothénique. 


\section{SUMMARY}

\section{EFFECT OF LIPOTROPIC FACTORS ON CHOLESTEROL METABOLISM}

\section{IN THE DUCKLING}

The effects were studied of dietary deficiencies of choline, inositol, pyridoxine and pantothenic acid on the distribution of cholesterol in the tissues (muscles, liver, heart, plasma) of ducklings. Increased concentrations of total cholesterol in muscles and heart of animals subjected to a dietary deficiency of choline were observed. Otherwise these animals presented no hepatic steatosis, no perosis and no increased concentrations of total cholesterol in plasma and liver. No specific increase in the concentrations of total lipids, or total and esterified cholesterol in the tissues was produced by dietary deficiency of pyridoxine in spite of the badly effect on the growth rate. The ratio in muscles, liver and heart of the concentration of cholesterol esters to the concentration of total cholesterol obtained in ducklings fed diets deficient in choline or pantothenic acid suggests that an increase in the rate of the cholesterol esterifying activity in plasma is probably instigated by these deficiencies.

\section{RÉFÉRENCES BIBLIOGRAPHIQUES}

Alary J. G., Demers J.-M., I965. L'influence du cholestérol exogène et de la choline dans un régime à base de gras de bœuf chez le caneton. Arch. Sci. Physiol., 19, 415-423.

Brot N., Lossow W. J., Chaikofr I. L., rg62. In vitro esterification of cholesterol by plasma : The effect of evisceration. J. Lipid Res., 3, 413-415.

Carbonneau R., Demers J.-M., I965a. Le complexe caséine-méthionine-choline dans la nutrition du caneton. Ann. Nutr. Aliment., 19, 59-70.

Carbonneau R., Demers J.-M., I965 b. La atéatose hépatique et la cholestérolémie chez le caneton domestique au cours d'une malnutrition protéique en régime orysé, Can. J. Comp. Med., 29, $271-278$.

Carbonneau R., Demers J.-M., 1965 c. Effects des régimes hyperlipidiques sur la surcharge grasse hépatique, les protéines et les lipides sanguins du caneton. Rev. Can. Biol., 75, I3I-I39.

Demers J.-M., I95I. Travaux de nutrition chez le canard domestique (Pékin blanc). Rev. d'Oka, 25, 6I-II9.

Field H. Jr, Swell P. E, Schools P. E. Jr, Treadwell C. R., ig6o. Dynamic aspects of cholesterol metabolism in different areas of the aorta and other tissues in man and their relationship to atherosclerosis. Circulation, 22, 547-558.

Folch J., Ascoli I., Lees M., Meath J. A., Iebaron F, N., 1951. Preparation of lipid extracts from brain tissue. J. Biol. Chem., 181, 833-84r.

Folch J., Lees M., Sloane-Stanley J. H., 1954. A simple method for the preparation of total pure lipid extracts from brain. Fed. Proc., 13, 209.

Genest P., I946. Technique de prélèvement de sang chez la poule par la ponction cardiaque. Can. J. Comp. Med., 10, 23-26.

Hegsted D. M., Stare F. J., I945. Nutrition studies with the duck I. Purified rations for the duck. J. Nutr., 30, 37-44.

Lofland H. B. Jr, Moury D. M., Hoffman C. W., Clarkson T. B., 1965. Lipid metabolism in pigeon aorta during atherogenesis. J. Lipid Res., 6, I I2-I I8.

Pearson S., Stern S., McGavach T. H., I954. A rapide acurate method for the determination of total cholesterol in serum. A nalytical Chem., 25, 813-8I4.

Shah S. N., Lossow W. J., Chaikoff I. L. I964. The esterification of cholesterol in vitro by rat plasma. I. Relative participation of triglycerides and phospholipids. II. Effect of snake venom. Biochem. Biophys. Acta, 84, I76-I8I.

Snyder F., Cornatzer W. F., Simonson G. E., r957. Comparative lipotropic and lipid phosphorylating effects of choline, betaine and inositol. Proc. Soc. Exp. Biol. Med., 96, 67o-672.

Sperry W. M., I935. Cholesterol esterase in blood. J. Biol. Chem., 111, 467-478.

Sugano M., Chinen I., WAda M., I966. Fatty acid specificity of cholesterol esterification in classes of serum lipoproteins of the chickens. J. Biochem., 60, 345-35r. 
Swaнn B., 1952. A new micromethod for the determination of total lipids in serum. Scand. Clin. Lab. Invest., 4, 247-248.

SwELL L., LAw L. D., I967. Heterogeneity in the composition of cholesterol esters synthesized by liver cell fractions of fasted and fed rats. Commun., 26, 206-2ro.

Wells I. C., Buckley J. M., r965. Lipid components of bile from choline-deficient rats. Proc. Soc. Exp. Biol. Med., 119, 242-243.

Wells I. C., Hogan J. M., 1968. Effects of dietary deficiencies of lipotropic factors on plasma cholesterol esterification and tissue cholesterol in rats. $J . N u t r ., 85,55-62$. 